\title{
Top-down fabricated silicon nanowires under tensile elastic strain up to $4.5 \%$
}

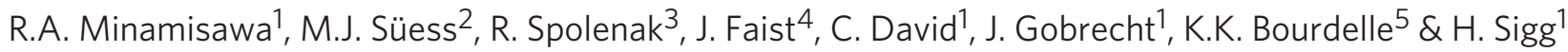

Strained Si nanowires are among the most promising transistor structures for implementation in very large-scale integration due to of their superior electrostatic control and enhanced transport properties. Realizing even higher strain levels within such nanowires are thus one of the current challenges in microelectronics. Here we achieve $4.5 \%$ of elastic strain ( $7.6 \mathrm{GPa}$ uniaxial tensile stress) in $30 \mathrm{~nm}$ wide Si nanowires, which considerably exceeds the limit that can be obtained using SiGe-based virtual substrates. Our approach is based on strain accumulation mechanisms in suspended dumbbell-shaped bridges patterned on strained Si-on-insulator, and is compatible with complementary metal oxide semiconductor fabrication. Potentially, this method can be applied to any tensile prestrained layer, provided the layer can be released from the substrate, enabling the fabrication of a variety of strained semiconductors with unique properties for applications in nanoelectronics, photonics and photovoltaics. This method also opens up opportunities for research on strained materials.

\footnotetext{
${ }^{1}$ Laboratory for Micro- and Nanotechnology, Paul Scherrer Institut, 5232 Villigen, Switzerland. ${ }^{2}$ Electron Microscopy, ETH Zürich, 8093 Zürich, Switzerland. ${ }^{3}$ Department of Materials, Laboratory for Nanometallurgy, ETH Zürich, 8093 Zürich, Switzerland. ${ }^{4}$ Institute for Quantum Electronics, ETH Zürich, 8093 Zürich, Switzerland. ${ }^{5}$ SOITEC, Parc Technologique des Fontaines, 38190 Bernin, France. Correspondence and requests for materials should be addressed to R.A.M. (email: renato.minamisawa@psi.ch) or to H.S. (email: hans.sigg@psi.ch).
} 
O ne of the great challenges to further improve the scaling down of silicon metal oxide semiconductor field effect transistors (MOSFETs) involves the increase of carrier mobility in the channel while maintaining electrostatic integrity ${ }^{1,2}$. The stringent electrostatic requirements have been met in ultra-short transistors by multigate geometry, where the optimum configuration is the nanowire (NW)-like architecture with a wrapped gate ${ }^{2,3}$. Mobility enhancement is in turn a very attractive option because it improves device performance beyond the benefits provided by scaling ${ }^{1}$. Mobility enhancement can be achieved by altering the properties of silicon through strain-induced manipulation of the band structure, which modifies the effective masses and phonon scattering within the channel ${ }^{4}$. For these reasons, silicon NWs with strained channels are promising candidates for the next generation of MOSFETs ${ }^{5}$.

The main strain-engineering approach currently pursued by the microelectronics industry is based on the deposition of stressor layers on top of transistors. Because the dimensions of current scaled transistors are becoming smaller than the required thickness of such an overlayer, it is difficult to implement this approach into smaller nodes while providing uniaxial tensile stress higher than the presently obtained $\sim 2 \mathrm{GPa}$ (corresponding to $\sim 1.2 \%$ strain) $)^{4,6}$. Indeed, the integration of strain levels higher than those currently available into silicon NWs is recognized as a major milestone for the front end silicon technology at smaller nodes?

An alternative way to produce strained silicon is to grow a thin pseudomorphic silicon cap over a strain-relaxed SiGe virtual substrate, which is a graded SiGe thick layer grown on a bulk silicon substrate to control the dislocation density ${ }^{8}$. The defect density and the biaxial tensile strain of the silicon cap depends on the germanium content of the SiGe layer; for example strained silicon with $0.8 \%$ strain ( $20 \%$ Ge content) has sufficiently low defect density of about $\sim 10^{4} \mathrm{~cm}^{-2}$. Strained silicon layers grown on higher germanium content buffer layers ${ }^{9}(\mathrm{Ge}>45 \%)$ could provide higher strain levels, but the drawback is that the dislocation density would increase to an unacceptable level.

Recently, silicon NWs with high uniaxial stress of $\sim 2 \mathrm{GPa}(\sim 1.2 \%$ strain) have been demonstrated by patterning suspended NWs on highly strained silicon on insulator $(\mathrm{SSOI})^{10}$. These substrates are fabricated by transferring the strained silicon layer from a virtual substrate to an oxidized silicon handle wafer via wafer bonding ${ }^{11}$. The NWs patterned on highly strained silicon layers are released from the buried $\mathrm{SiO}_{2}$ by wet chemical etching, relaxing the free surfaces while preserving the initial longitudinal strain. Up to two times enhancement in transconductance has been reported for transistors built with such highly strained NWs in respect to those made on conventional silicon substrate ${ }^{10}$.

Micro electromechanical system loading has been proposed as an alternative to produce very high longitudinal strain in vapour liquid solid grown $\langle 111\rangle$ oriented silicon $\mathrm{NWs}^{12}$. In this method, the NW is longitudinally stretched by releasing a prestrained contact cantilever. Although this technique has produced silicon NWs with strain up to $\sim 3.5 \%$, it is still limited for very large-scale integration (VLSI) due to the intrinsic doping of the NW, the use of gold catalysts, the non ideal crystal orientation, the dimensional variability, the large size of the micro electromechanical system and most importantly, the difficulty of controlling the strain. Preconditions for Si microelectronic industry are strict and require compatibility to VLSI substrates, processes using few processing steps and flexibility to a variety of layered systems.

In this work, by patterning and releasing dumbbell-shaped bridges from thin biaxial tensile SSOI substrates, we extend the above approaches to fabricate high-quality suspended silicon NWs with longitudinal strain up to $\sim 4.5 \%$ ( $\sim 7.6 \mathrm{GPa}$ uniaxial tensile stress). We use the word 'bridge' to describe an entire structure, including a narrow central NW together with its supporting suspended pads. We utilize a fabrication process that is fully compatible with mainstream complementary metal oxide semiconductor (CMOS) technology. Our bridges are fabricated from SSOI substrates, but the concept can be generalized and used for any tensile strained layer, including free standing defect free $\mathrm{Si} / \mathrm{SiGe}$ heterostructure membranes ${ }^{13}$. We show that the strain can be controlled accurately by designing the dimensions of the bridge, producing a uniform longitudinal strain in the NW. Further, we demonstrate the extension of the concept to multi-NWs.

\section{Results}

Strain enhancement in dumbbell-shaped $\mathrm{Si}$ nano structures. Necked samples are generally used in mechanical tensile tests to control the localisation of the stress and, eventually, the break point ${ }^{14}$. Here dumbbell-shaped structures are used to increase the strain of equibiaxially tensile-stressed SSOI. We reduce the cross-sectional area of the samples, thereby concentrating the tension to the central nanometre sized part of the structure. The starting strain in the equibiaxially tensilestressed silicon layer amounts to $\varepsilon_{0} \sim 0.8 \%$ as verified by micro Raman measurements. We use SSOI with an intermediate strain because its defect density is lower than that of highly strained SSOI.

We patterned dumbbell-shaped structures with different dimensions on $13 \mathrm{~nm}$ thick SSOI followed by etching the buried $\mathrm{SiO}_{2}$. The bridges were patterned in the $\langle 110\rangle$ orientation, which, for strain levels higher than $1 \%$, induces higher electron mobility than biaxial $\operatorname{strain}^{15}$. The five dimensions displayed in Fig. 1a classify the resulting suspended bridges: the central NW length $A$, the overall bridge length $B$, the NW width $a$ and the pad width $b$. An additional parameter shown in Fig. 1a is the length $L$ etched underneath the buried oxide, beyond the outer frame of the structure. The released bridges relax in the transverse directions ${ }^{16}$ while transferring longitudinally strain from the two pads to the NW located at the bridge centre. Assuming a constant silicon layer thickness, the cross-sectional dependency of the stress distribution used to determine the strain enhancement in the NWs can be reduced to a dependency on the length ratio, $(B-A) / A$, and the width ratio, $b / a$. The strain enhancement $\varepsilon_{x} / \varepsilon_{0}$, defined as the ratio of strain along the NW $\varepsilon_{x}$ divided by the initial strain $\varepsilon_{0}$ of the layer, is related to the dimensions of the bridge according to (see details in Supplementary Methods),

$$
\varepsilon_{x} / \varepsilon_{0}=C \times[1+A /(B-A)] /[A /(B-A)+a / b],
$$

where $C$ is a correction factor to account for the influence of etching underneath the frame. The factor $C$ is discussed later. This expression follows readily from the consideration of a local minimum of the elastic energy, which is achieved by elastic relaxation of the pads (reduction of the free energy) at expense of expansion of the inner NW part, which increases the elastic energy therein. Note that equation (1) is purely a geometrical relation, independent of material properties.

Micro Raman experiments and finite element methods. Micro Raman measurements along a suspended bridge with a $30 \mathrm{~nm}$-wide NW give clear evidence that the strain in the NW is enhanced by the elastic relaxation of the contact pads (Fig. 1b). A typical Raman spectrum of the SSOI layer features two peaks at $520.0 \mathrm{~cm}^{-1}$ (corresponding to bulk cubic silicon), and at $514.5 \mathrm{~cm}^{-1}$ (corresponding to the $0.8 \%$ biaxially tensile-strained Si layer). In the contact pad of the suspended bridge, the strained silicon peak shifts towards that of the cubic silicon as an indication of strain relaxation, whereas in the NW the peak shifts towards lower wavenumbers indicating a strain increase. The Raman peak shift $\Delta \omega$ was determined by deconvolution of the strained and unstrained cubic silicon signals extracted from the line scan measurements (Fig. 1c). Within the resolution of our Raman instrument, the values of strain are constant along the NW yielding a value of about $13.9 \mathrm{~cm}^{-1}$. The strain $\varepsilon_{x}$ along 
a
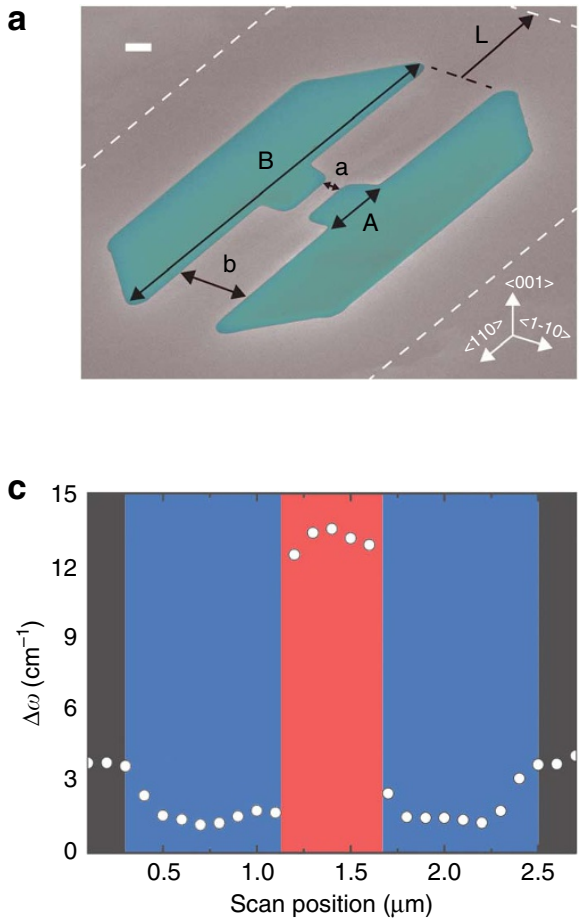

b

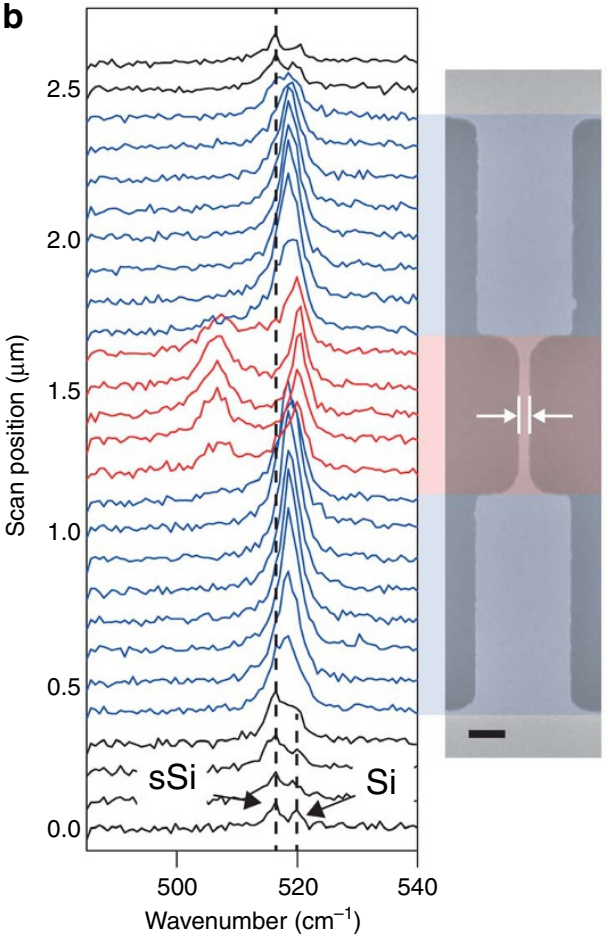

Figure 1 | Microscopy images of the NWs and Raman spectroscopy mapping. (a) Coloured SEM picture of a suspended strained Si dumbbell bridge with a $200 \mathrm{~nm}$ narrow and $13 \mathrm{~nm}$ thin NW. The dimensions $A, B$, $a$, and $b$ refers to the NW length, the bridge length, the NW width and the pad width, respectively. The dimension $L$ corresponds to the length of the etching underneath the outer frame, represented by the white dashed line. The scale bar represents $300 \mathrm{~nm}$. (b) Raman line scan measurement of a bridge with $A=0.6 \mu \mathrm{m}, B=2.4 \mu \mathrm{m}, a=30 \mathrm{~nm}$ and $b=360 \mathrm{~nm}$. A SEM picture of the measured bridge is displayed on the right. The Si peak from the substrate is located at $520.0 \mathrm{~cm}^{-1}$, whereas the peaks from the biaxial-strained layer and the uniaxially strained NW are at 514.5 and $506.1 \mathrm{~cm}^{-1}$, respectively. The red and blue shaded areas indicate the NW and pad, respectively. The black scale bar represents $100 \mathrm{~nm}$, whereas the distance between the white arrows indicates that the wire has $30 \mathrm{~nm}$ width. (c) Raman shift of the strained Si peak in respect to relaxed $\mathrm{Si}$ as a function of the longitudinal position along the bridge. The red and blue shaded areas indicate the position of NW and pad, respectively.

$\langle 110\rangle$ direction is calculated from the Raman shift $\Delta \omega$ through the following semi-empirical equation for uniaxially stressed $\mathrm{Si}^{17}$ :

$$
\Delta \omega=-1.93 \mathrm{~cm}^{-1} \times \varepsilon_{x} /\left[\left(S_{11}+S_{12}\right) / 2+S_{44} / 4\right]=-3.27 \mathrm{~cm}^{-1} \times \varepsilon_{x}
$$

where $S_{11}, S_{12}$, and $S_{44}$ are the silicon compliance tensor entries ${ }^{17}$. From equation (2) the longitudinal strain in the $30 \mathrm{~nm}$-wide NW displayed in Fig. $1 \mathrm{~b}$ is about $4.0 \%$.

A detailed analysis of the strain components in the silicon bridges is found by finite element simulations (Fig. 2a,b), which confirms that the longitudinal tensile strain $\varepsilon_{x}$ is homogeneously distributed in the NW and amounts $\sim 4.0 \%$, whereas it partially relaxes to about $0.4 \%$ in the contact pads. The transverse strain components $\varepsilon_{y}$ and $\varepsilon_{z}$ of the NW are negative (Fig. 2b) as expected.

The impact of the etching underneath the frame on the strain of the NW estimated through finite element simulations is quantified in Fig. 2c, which shows bridges with constant cross-sectional ratio $b / a=5$ for different $b$ and $a$ values. We observe experimentally and in simulations that extending the under etching length $L$ (Fig. 1a) results in a substantial increase of the strain in the wire. The effect is weakly dependent on the widths of the bridge at constant crosssectional ratio (Fig. 2c). An enhancement also occurs for the case where $a$ approaches $b$, when, strictly speaking, no dumbbell-like structure is formed (see Supplementary Discussion). Instead, the substrate-released bridge resembles a dumbbell with $b>>a$, which led us to approximate the correction factor in equation (1) by $C=(B+2 L) / B$, where $L$ is experimentally determined by scanning electron microscopy (SEM) inspection (Supplementary Discussion).
Strain dependence on structural dimension. We demonstrate that our approach enables fine tuning of the applied strain in the NWs by varying the dimensions of the bridge components, that is, lengths and widths of both pads and wire. Micro Raman spectra of silicon NWs with widths of $a=125,375$ and $725 \mathrm{~nm}$ from bridges with fixed dimensions $A=0.6 \mu \mathrm{m}, B=2.4 \mu \mathrm{m}$ and $b=1.0 \mu \mathrm{m}$ are shown in Fig. 2 d. The longitudinal strain increases for narrower wires as a consequence of the reduced cross section, that is, increased force per area. The decrease of signal strength observed in the spectra of smaller NWs stems from the reduced overlap between the nanostructure and the laser spot (approximately $400 \mathrm{~nm}$ diameter).

Figure $3 \mathrm{a}$ shows the strain of NWs as a function of the pad width for wire widths ranging from 30 to $175 \mathrm{~nm}$. The overall length of the bridge is $B=2.4 \mu \mathrm{m}$ and the length of its NW component is $A=0.6 \mu \mathrm{m}$. The strain increases up to $4.5 \%$ by increasing the pad width or decreasing the NW width. In addition, increasing the length $L$ of the etching underneath the outer frame from 600 to $750 \mathrm{~nm}$ for a bridge with $a=30 \mathrm{~nm}$ results in 1.5 times increase in strain. The results obtained from equation (1) using the above dimensions are also plotted.

The strain in NWs with larger widths ranging from 125 to $975 \mathrm{~nm}$ and fixed pad width of $b=1.5 \mu \mathrm{m}$ is shown in Fig. 3b. The NW length was fixed to $A=0.6 \mu \mathrm{m}$, whereas the bridge length varied from $B=2.4$ to $4.4 \mu \mathrm{m}$. In this case, the strain decreases by increasing the NW width $a$. The strain in the NW increases in respect to the biaxial case even as $a$ approaches $b$. The strain under this condition is found to be $\sim 1.5 \%$ uniformly distributed along the entire bridge, which is consistent with equation (1) taking $L$ as $\sim 750 \mathrm{~nm}$. 
a
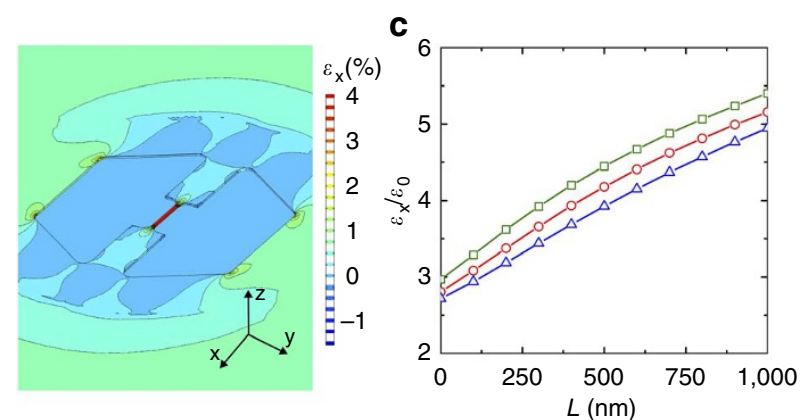

b
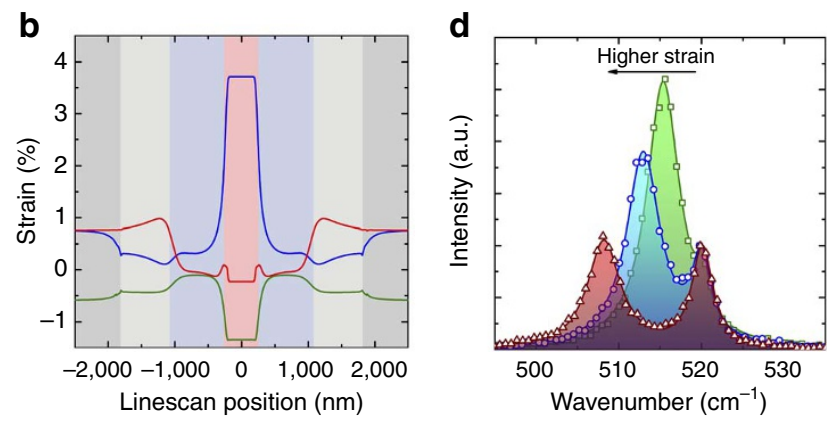

Figure $\mathbf{2}$ | Finite element strain simulations and Raman measurements.

(a) 3D finite element simulation of the longitudinal strain component $\varepsilon_{x}$ of a bridge with dimensions as shown in Fig. $1 \mathrm{~b} ; A=0.6 \mu \mathrm{m}, B=2.4 \mu \mathrm{m}$, $a=30 \mathrm{~nm}, b=360 \mathrm{~nm}$ and $L=600 \mathrm{~nm}$, where $A, B, a$ and $b$ refers to the NW length, the bridge length, the NW width and the pad width, respectively, and $L$ to the under-etched length. (b) Simulated strain components along $x=\langle 110\rangle$ (blue), $y=\langle 1-10\rangle$ (green) and $z=\langle 001\rangle$ (red) as a function of the line scan position along the length of the bridge displayed in (a). The shaded areas represent the NW (red), pads (blue), under-etched region (light grey) and biaxially stressed layer (dark grey). (c) Strain enhancement in the NW as a function of the underetching length $L$, for a bridge with $b / a=5, B / A=4$ and $B=2,400 \mathrm{~nm}$. The simulation was performed for wires with 50 (green square), 100 (red circle) and 200 (blue triangle) nm widths. (d) Raman spectra of Si NWs with widths $a=125$ (green square), 375 (blue circle) and 725 (red triangle) $\mathrm{nm}$ from bridges with $A=0.6 \mu \mathrm{m}, B=2.4 \mu \mathrm{m}$ and $b=1.0 \mu \mathrm{m}$.
The strain enhancement versus the cross section ratio for bridges with different $a$ and $b$ and with different length ratios is shown in Fig. 3c. The enhancement amounts up to six times beyond the starting strain and is limited only by fracture of the bridges at strain above $4.5 \%$. We found this limit to be independent of the NW width. The experimental data closely match the prediction of equation (1), which indicates that both the width and strain of the fabricated NWs can be controlled by the design of the bridge. Thus, these data provide strong support for the generality of our approach.

Strain enhancement in multi-NWs. Furthermore, we extend our approach to the fabrication of strained silicon multi-NWs. MultiNW silicon transistors have been reported as promising building blocks for high-performance logic inverters, fast ring oscillators and demultiplexers ${ }^{18,19}$. These devices offer excellent electrostatic control, and higher drive current than those with single wires ${ }^{20}$. The SEM images of such multi-NWs and the respective Raman spectra are shown in Fig. 4. The strain increases with decreasing the number of wires, such that for single, double and triple wires, the strain is $3.3 \%, 3.1 \%$ and $2.8 \%$, respectively. Linescan measurements show that every individual NW experiences the same strain as its neighbours. The equal distribution of the strain within the array indicates that the strain enhancement does not generate new dislocations.

\section{Discussion}

The mechanical stability and precise positioning control of such highly strained Si NWs in the substrate allows its robust integration into standard transistors. A feasible approach would be, for example, to employ the well-established gate-all-around architeture ${ }^{10,21}$. In order to realize this design, we propose employing a gate last process in order to avoid strain relaxation, where activation of source and drain is performed before patterning, and where low temperature conformal deposition of high- $\kappa$ gate oxide and metal gate is achieved using atomic layer deposition ${ }^{22}$. Although in the present form, the strained NWs are much larger than what is required to VLSI, strain enhancement is solely dependent on the bridge dimensions such that the structures can be scaled down to the limits that lithography methods provide. Yet, our strain technique does not require stressor layers, which may contribute to decrease the contacted gate pitch. Finally, our approach could be used for logic device applications a

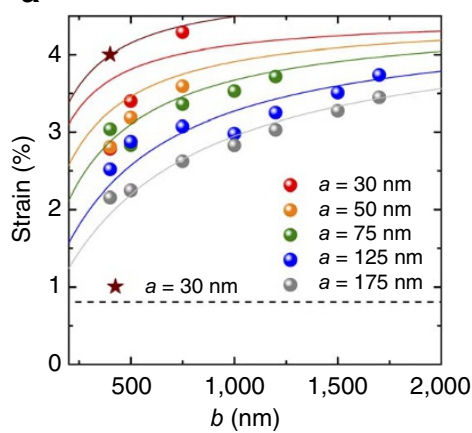

b

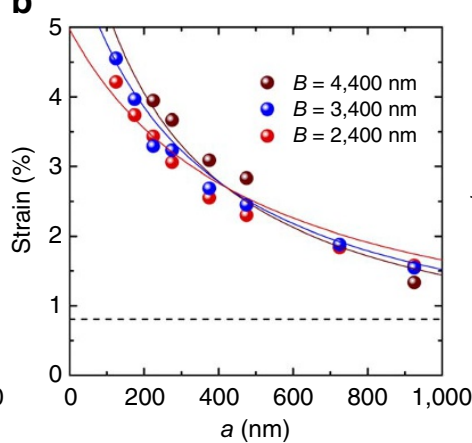

c

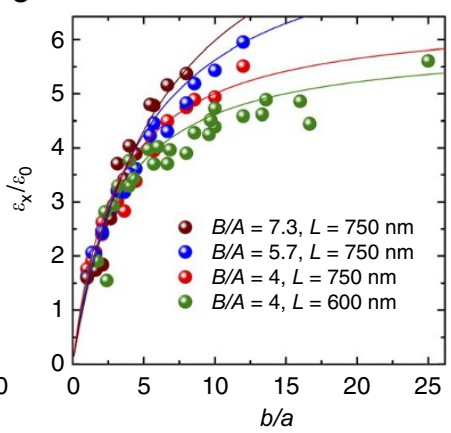

Figure 3 | Strain dependence on the dimensions of the nanobridges. (a) Longitudinal strain of the NW versus the pad width $b$ for $B=2.4 \mu \mathrm{m}$ and $A=0.6 \mu \mathrm{m}$, and different NW widths $a . A, B, a$ and $b$ refers to the NW length, the bridge length, the NW width and the pad width, respectively, and $L$ to the under-etched length. The under-etched length $L$ is $600 \mathrm{~nm}$ for the circle symbol keys, whereas $750 \mathrm{~nm}$ for the star symbol key. The strain of the biaxially SSOI is plotted for reference (dashed line). (b) Strain as a function of the NW width $a$ for a constant pad width $b=1.5 \mu \mathrm{m}$ and $L=750 \mathrm{~nm}$. The wire length is $A=0.6 \mu \mathrm{m}$, whereas the bridge length was varied from $B=2.4$ to $4.4 \mu \mathrm{m}$. The strain of the biaxially SSOI is plotted for reference (dashed line). (c) Strain enhancement versus the ratio $b / a$ for NWs with different $b$ and $a$ values. The curved lines were obtained from equation. (1), whereas the points represent experimental measurements. 


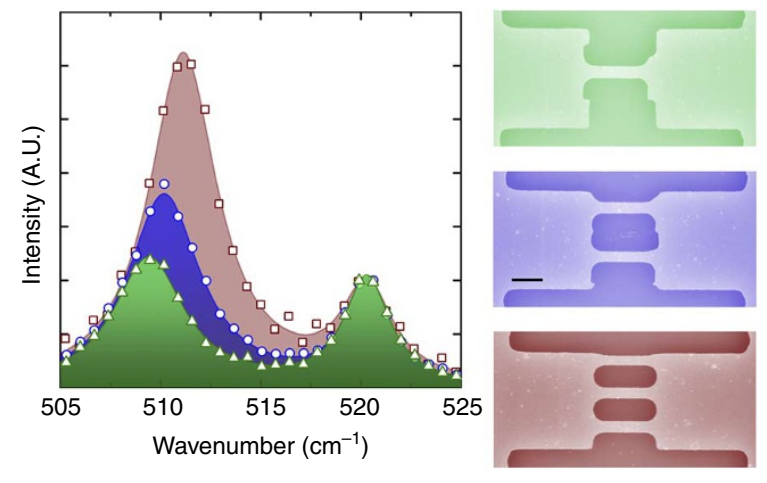

Figure 4 | Microscopy images and Raman measurements of multi-NWs. False-coloured SEM images (right) of single (green), double (blue) and triple multi-NWs (brown). The scale bar represents $300 \mathrm{~nm}$. Raman spectra of single (green triangle), double (blue circle) and triple multiNWs (brown square) (left) with strain values of 3.34\% (green), 3.09\% (blue) and $2.82 \%$ (brown), respectively.

that require low-power MOSFETs but not aggressive scaling such as cellphone circuits. In addition, at 7.6 GPa uniaxial stress, the $\mathrm{Si}$ bandgap reduces to about $0.5 \mathrm{eV}$ as verified by $6 \times 6 \mathrm{kp}$ simulations including deformation potentials as provided by the software package Nextnano, which makes our approach very attractive to ultra low power band-to-band tunnelling field effect transistors ${ }^{23}$.

From a materials point of view our results are also unique as they demonstrate extremely high uniform strain along $\mathrm{x}, \mathrm{y}$ and $\mathrm{z}$ orthogonal directions (see Fig. 2b) in contrast to those found in a NW bending geometry ${ }^{21}$. The strain levels are still lower than the theoretical tensile strength of a brittle material, which is expected to be $\sim E / 10$, where $E$ is the Young's modulus. These levels under uniform tensile stress have only been observed so far by Zhu et $a{ }^{24}$ for epitaxialy grown Si nanowhisker with diameters of about $15 \mathrm{~nm}$, strained using actuators. For the dimensions of our bridges $(\geq 30 \mathrm{~nm})$, we achieved comparable tensile strength, though our NWs were fabricated using a top down approach. This fracture strength $(\sim 7.6 \mathrm{GPa})$ is also equivalent to those obtained in top down fabricated Si NWs from defect free SOI using external actuators ${ }^{25}$.

To summarize, we have demonstrated record strains in silicon NWs without actuators or stressor layers but rather by transferring the strain within the suspended structure. It is noteworthy that the achieved strain is higher than those achievable by conventional methods using virtual substrates. Moreover, the method presented here can be applied to any pseudomorphically grown tensilestrained heterostructure material system as it depends purely on geometrical factors. As an example, apart from the application to silicon NWs, this approach could very well be applied to micrometre thick strained Ge layers for photonic applications ${ }^{26}$.

\section{Methods \\ Fabrication of the strained Si suspended constricted structures. The starting material for the NW fabrication consisted of $13 \mathrm{~nm}$ thick equibiaxial tensile- stressed silicon on insulator layer on top of a $145 \mathrm{~nm}$ buried oxide layer. Details on the fabrication process and the properties of the SSOI material are described elsewhere ${ }^{11}$. Raman measurements indicated a positive strain of $\varepsilon=0.8 \%$ in the SSOI layer. Electron beam lithography and reactive ion etching using low-energy $\mathrm{SF}_{6}$-based plasma were employed to define the dumbbell bridges with different dimensions oriented along the [110] crystal direction. The electron beam litho- graphy resist was removed using Piranha solution. The bridge dimensions were verified by SEM. The buried $\mathrm{SiO}_{2}$ was etched by directly immersing the samples into buffered hydrofluoric acid (HF) (7:1) during 4-6 min, followed by rinsing in deionized water for $10 \mathrm{~min}$}

Raman characterization of strain in the nanostructures. Raman spectroscopy was performed using a WITec CRM200 instrument, with a $532 \mathrm{~nm}$ excitation and spectral resolution of $0.3 \mathrm{~cm}^{-1}$. Spectra were collected in backscatter configuration using a X100 magnification optic. All the spectra feature a peak at $520.0 \mathrm{~cm}^{-1}$, corresponding to the relaxed $\mathrm{Si}$ substrate, and a peak found at a position between 505.0 and $520.0 \mathrm{~cm}^{-1}$, corresponding to strained $\mathrm{Si}$. The relative Raman shift used for the calculation of strain was retrieved by fitting the spectra with two Lorentzians functions and extracting the difference between the peak positions. The s.d. of the peak position is found to be approximately $0.15 \mathrm{~cm}^{-1}$.

\section{References}

1. Ieong, M., Doris, B., Kedzierski, J., Rim, K. \& Yang, M. Silicon device scaling to the sub-10 nm regime. Science 306, 2057-2060 (2004).

2. Chau, R., Doyle, B., Datta, S., Kavalieros, J. \& Zhang, K. Integrated nanoelectronics for the future. Nat. Mat. 6, 810-812 (2007).

3. Ferry, D. K. Nanowires in nanoelectronics. Science 319, 579-580 (2008).

4. Chu, M., Sun, Y., Aghoram, U. \& Thompson, S. E. Strain: a solution for higher carrier mobility in scaled MOSFETs. Annu. Rev. Mater. Res. 39, 203-229 (2009).

5. Auth, C. et al. A $22 \mathrm{~nm}$ high performance and low-power CMOS technology featuring fully-depleted trigate transistors, in Symposia on VLSI Technology and Circuits. 131-132 (2012).

6. Thompson, S. E., Sun, G., Choi, Y. S. \& Nishida, T. Uniaxial process induced strained Si: extending the CMOS roadmap. IEEE Trans. Electron Devices 53, 1010-1020 (2006).

7. International Technology Roadmap for Semiconductors 2010 report. Available at http://www.itrs.net/home.html.

8. Currie, M. T., Samavedam, S. B., Langdo, T. A., Leitz, C. W. \& Fitzgerald, E. A. Controlling threading dislocation densities in $\mathrm{Ge}$ on $\mathrm{Si}$ using graded SiGe layers and chemical-mechanical polishing. Appl. Phys. Lett. 72, 1718-1720 (1998).

9. Paul, D. J. Si/SiGe heterostructures: from material and physics to devices and circuits. Semicon. Sci. Tech. 19, R75-R108 (2004).

10. Hashemi, P., Gomez, L. \& Hoyt, J. L. Gate-all-around n-MOSFETs with uniaxial tensile strain-induced performance enhancement scalable to sub-10-nm nanowire diameter. IEEE Electron Device Lett. 30, 401-403 (2009).

11. Ghyselen, B. et al. Engineering strained silicon on insulator wafers with the Smart Cut $^{\mathrm{Tm}}$ technology. Solid State Electron. 48, 1285-1296 (2004).

12. Lugstein, A., Steinmair, M., Steiger, A., Kosina, H. \& Bertagnolli, E. Anomalous piezoresistance effect in ultra strained silicon nanowires. NanoLetters 10, 3204-3208 (2010).

13. Roberts, M. M. et al. Elastically relaxed free-standing strained-silicon nanomembranes. Nat. Mat. 5, 388-393 (2006).

14. Bauman, J. T. Fatigue, Stress, and Strain of Rubber Components: Guide for Design Engineers (Hanser Publication, Cincinnati, 2008).

15. De Wolf, I., Maes, H. E. \& Jones, S. K. Stress measurements in silicon devices through Raman spectroscopy: bridging the gap between theory and experiment. J. Appl. Phys. 79, 7148-7156 (1996).

16. Minamisawa, R. A. et al. Elastic strain and dopant activation in ion implanted strained Si nanowires. J. Appl. Phys. 108, 124908 (2010).

17. Chen, F. et al. Conduction band structure and electron mobility in uniaxially strained Si via applied strain in nanomembranes. J. Phys. D: Appl. Phys. 44, 325107 (2011).

18. Friedman, R. S., McAlpine, M. C., Ricketts, D. S., Ham, D. \& Lieber, C. M. High-speed integrated nanowire circuits. Nature 434, 1085 (2005).

19. Beckman, R., Johnston-Halperin, E., Luo, Y., Green, J. E. \& Heath, J. R. Bridging dimensions: demultiplexing ultrahigh-density nanowire circuits. Science 310, 465-468 (2005).

20. Gunawan, O. et al. Measurement of carrier mobility in silicon nanowires. NanoLetters 8, 1566-1571 (2008).

21. Moselung, K. E. et al. The high mobility bended n-channel silicon nanowire transistor. IEEE Transactions on Electron Devices 57, 866-876 (2010).

22. Colinge, J.- P. FinFETs and other multi-gate transistors (Springer, Cambridge, 2008).

23. Smith, J. T. et al. Silicon nanowire tunneling field-effect transistor arrays: improving subthreshold performance using excimer laser annealing. IEEE Transact. Electron Devices 58, 1822-1829 (2011).

24. Zhu, Y., Xu, F., Qin, Q., Fung, W. Y. \& Lu, W. Mechanical properties of vapor-liquid-solid synthesized silicon nanowires. NanoLetters 9 , 3934-3939 (2009).

25. Bhaskar, U. et al. Onchip tensile testing of nanoscale silicon freestanding beams. J. Mater. Res. 27, 571-579 (2011).

26. Liu, J., Sun, X., Camacho-Aguilera, R., Kimerling, L. C. \& Michel, J. Ge-on-Si laser operating at room temperature. Optics Lett. 35, 679-681 (2010).

\section{Acknowledgements}

We thank the financial support by the Swiss National Science Foundation (SNF Project- $n^{\circ} .130181$ ) and the Swiss Priority Program nano-tera.ch. We also thank G. Schiefler for his contribution on predevelopments of the strain multiplication technique and K. Bedner and C. Spreu for assistance with e-beam lithography and RIE etching, respectively. R.A.M. thanks Prof. L.R. Holland and Prof. R.L. Zimmerman 
for reviewing the manuscript. R.A. Minamisawa and M.J. Süess contributed equally to this work.

\section{Author contributions}

R.A.M. fabricated the Si bridges; R.A.M. and M.J.S. performed Raman measurements; M.J.S. carried out finite element simulations; H.S., R.S., C.D. and J.G. collaborated on equipments, H.S., R.S., and J.F. collaborated on the design of the experiment; K.K.B. provided the SSOI wafers; R.A.M. and H.S. wrote the paper draft. H.S. supervised the experiments and coordinated the data interpretation.

\section{Additional information}

Supplementary Information accompanies this paper at http://www.nature.com/ naturecommunications

Competing financial interests: The authors declare no competing financial interests.

Reprints and permission information is available online at http://npg.nature.com/ reprintsandpermissions/

How to cite this article: Minamisawa, R.A. et al. Top-down fabricated silicon nanowires under tensile elastic strain up to 4.5\%. Nat. Commun. 3:1096 doi: 10.1038/ncomms2102 (2012). 